\title{
LA LITERATURA EN EXPANSIÓN. INTERMEDIALIDAD Y TRANSMEDIALIDAD EN EL CRUCE ENTRE LA LITERATURA COMPARADA, LOS ESTUDIOS CULTURALES Y LOS NEW MEDIA STUDIES ${ }^{1}$
}

Domingo SÁNCHEZ-MESA

Universidad de Granada dsanchez@ugr.es

Jan BAETENS

KU Leuven jan.baetens@kuleuven.be

\section{1}

\section{Introducción: la Literatura Comparada en movimiento}

Al menos desde finales de los años 70, el campo tradicional de la Literatura Comparada se ha caracterizado por dos cambios principales ${ }^{2}$. Uno de ellos descansa en la idea de que la Literatura Comparada como disciplina basada en el componente lingüístico se ha vuelto más compleja. Y ello no solo porque el lenguaje haya dejado de considerarse «simplemente» verbal para pasar a convertirse en una realidad multi- o polimodal, sino también, y de forma más relevante, porque la idea de lenguaje o incluso de texto se ha vuelto subordinada a la idea de medio, un concepto que ya no se restringe a elementos lingüísticos o verbales. Ya la comparación interartística recibió una atención y un espacio significativo dentro de la Literatura Comparada, un cambio que generó más espacio para un enfoque más basado en la categoría de medio para el estudio comparativo de la literatura. La Literatura Comparada, en otras palabras, ya no se limita a las relaciones textuales sino que integra también en su objeto de estudio elementos propios de medios no estrictamente verbales.

\footnotetext{
${ }^{1}$ Este artículo es una versión ampliada y completamente revisada del titulado «Literature at the Expanded Field: Interrmediality at the Crossroads of Literary Theory and Comparative Literature», publicado en Interfaces. Image-Text Langages, 36, 2015, pp. 289-304. Ha sido escrito en el marco y con el apoyo del proyecto I+D «Narrativas Transmediales: Nuevos Modos de Ficción Audiovisual, Comunicación Periodística y Performance en la Era Digital», nº ref. CSO201347288-P (Univ. de Granada), del Programa Estatal de Fomento de la Investigación Científica y Técnica de Excelencia (Ministerio de Economía y Competitividad).

${ }^{2}$ Los distintos informes sobre el estado de la disciplina que fueron encargados por la ACLA (Levin, 1965; Green, 1975; Bernheimer, 1993; Saussy, 2004) registran esta evolución y permanente auto-cuestionamiento.
} 
La creación de programas de estudios sobre imagen y palabra («Word \& Image») así como la institucionalización de este nuevo campo a través de organizaciones profesionales tales como la IAWIS - International Association of Word and Image Studies ${ }^{3}$ - fueron las consecuencias lógicas, y los aceleradores, de esta evolución. Sin embargo, observaciones parecidas y, tal vez, incluso más radicales podrían hacerse desde el dominio de la Narratología, cuya organización profesional más influyente, ISSN - The International Association for the Study of Narrative- ha ampliado explícitamente su área de investigación a todo tipo de narrativas ya sean verbales o no verbales ${ }^{4}$.

El texto y, por extensión, la literatura ya no son el centro exclusivo del universo comparativo, cuyo núcleo está ahora compuesto por nociones tales como medio y adaptación.

En segundo lugar, la rápida evolución de la Literatura Comparada hacia la comparación interartística y, ocasionalmente, hacia los estudios comparados de medios (Comparative Media Studies), ha tenido lugar precisamente allí donde la Teoría de la Literatura ha permeado más intensamente dicho cambio ${ }^{5}$, convirtiéndose, de esta manera, en un campo de batalla terminológico que continúa movilizando grandes cantidades de energía e inventiva. Términos tales como intermedialidad, remediación, hipermediación o transmedialidad ${ }^{6}$, entre otros, han aparecido en los debates sobre literatura comparada, y parece evidente que el diálogo y la discusión en torno a ellos va a prolongarse durante algún tiempo. En este sentido, una clarificación y simplificación terminológicas son necesarias para poder progresar en dicho debate y ser capaces de tratar otros problemas cabalmente.

\section{Por una modesta propuesta terminológica}

En aras de la citada necesidad de clarificación en los debates terminológicos ya señalamos la necesidad (Sánchez-Mesa, 2011: 2012) de una reducción más bien drástica de la terminología existente en una oposición binaria, la que confronta provisionalmente las palabras o conceptos intermedial y transmedial $^{7}$. Siendo conscientes de las limitaciones de dicha simplificación, esperamos sin embargo

\footnotetext{
${ }^{3}$ Véase www.iawis.org (IAWIS fue creada en 1987).

${ }^{4}$ Véase http://narrative.georgetown.edu/ (su primer congreso anual tuvo lugar en 1986) o la inclusión de voces como «Narrations indexzw various media» (M.-L. Ryan) o «Narration in Film« (Markus Khun y Johan Schimdt) en el Living Handbook of Narratology (version wiki del Handbook of Narratology, Walter de Gruyter 2009, disponible en http://www.lhn.uni-hamburg.de).

${ }^{5}$ La Semiótica y la Retórica en el caso del Grupo «Mi», la Narratología en el caso de M.-L. Ryan y Grishakova, o la desconstrucción en el caso del pictorial turn acuñado por W.J.T. Mitchell (1988, 1994). En España, los libros de Antonio Monegal (1998), José Luis Brea (2007, 2008), José A. Pérez Bowie (2010) o Anxo Abuín (2013) son buenos exponentes de esta simbiosis.

${ }^{6}$ Conviene recordar la conveniencia de utilizar el término transmedia como adjetivo más que como sustantivo, tendencia generalizada en el ámbito académico y cultural anglosajón (toda innovación va siendo inmediatamente calificada de «transmedia»), ya que, como señaló Geoffrey Long (2008), en principio, es la presencia de un relato lo que determina el sintagma «transmedia storytelling». Otra cosa es que, como empieza a vaticinar la misma M.-L. Ryan (en prensa), «el transmedia» empiece a configurarse como un medio en sí mismo (capacidad de expresión + canal y soporte de distribución de contenidos).

${ }^{7}$ Esta oposición, que nosotros entendemos de forma dialógica, destacaba la diferencia entre dos modos teóricos de describir los fenómenos de transferencia entre y a través de los medios de masas tradicionales, por un lado, y entre estos y los medios digitales e interactivos por el otro. Por un lado, el transmedia storytelling, con un concepto fundamentalmente cognitivo
} 
que los beneficios prácticos de esta apuesta sean más importantes que las pérdidas ${ }^{8}$. De lo que se trata aquí no es de determinar si la literatura, el cine, la televisión o la novela gráfica son más o menos intermediales o transmediales - de hecho reclamamos que tanto la intermedialidad como la transmedialidad pueden ser rasgos propios de la forma literaria (no necesariamente de cualquier literatura pero sí de una parte importante de ella) — sino de desarrollar un modelo tan simple como sea posible para discutir temas literarios en el contexto contemporáneo más amplio de la cultura multimedia digital. Con objeto de permitir un entendimiento correcto del transmedia storytelling, introduciremos otras dos nociones, la demediación (concepto que tomamos prestado de Garrett Stewart, 2011) y la serialización (en el sentido en el que la ha trabajado Matthieu Letourneux, 2014). No obstante, las nociones gemelas de intermedialidad y transmedialidad son esenciales para nuestra argumentación en este ensayo.

2. 1. La intermedialidad: La teoría de los medios ya dejó claro que cada medio, sea verbal o no verbal, es por definición plural (Mitchell, 2005; Baetens, 2014). No hay, por tanto, medios «puros». Cada medio, incluyendo la escritura, es intermedial y dicha intermedialidad tiene dos dimensiones. Por un lado, cada medio se encuentra directa o indirectamente en contacto con otros medios y es influenciado así como transformado por ellos en el paisaje mediático más amplio. Primero Marshall McLuhan (1964) y luego Jay David Bolter y Richard Grusin (1999) han insistido en el hecho de cada medio es de hecho el resultado de una remediación debido a su conflicto con otros medios. Por otro lado, cada medio es a su vez una mezcla de diversos medios (en la escritura, por ejemplo, hay visualidad al igual que oralidad, pero también se da el tacto e incluso el olor). En la práctica, sin embargo, el uso del término intermedialidad se reduce a aquellos casos en que se combinan diferentes tipos de signos, por ejemplo palabras e imágenes ${ }^{9}$.

de lo narrativo como núcleo ontológico de la subjetividad posmoderna, implica una perspectiva que tiende a orientarse a describir la producción de las dinámicas del branded content y de las oportunidades de negocio en la cultura de la convergencia (Jenkins, 2003, 2006; Long, 2007, Scolari, 2009; Pratten 2011, 2015), donde dicho sujeto se convierte básicamente en un «prosumidor» de «mundos ficcionales transmediales» (Jenkins, 2003, 2006, 2009; Klastrupy Pajares Tosca, 2004; Pratten, 2011; Scolari, 2009, 2013). Por otro lado, nos encontramos con otra perspectiva o actitud crítica que podemos llamar «diferencial» o «dialógica», más sensible a la fricción, los anacronismos y al conflicto tanto en el nivel inter- como intramedial, más atenta a la especificidad de cada medio y sus prácticas culturales, así como a sus dimensiones discursivas y, en consecuencia, más capaz de evitar la reducción de la experiencia cultural y artística a la abstracción de los contenidos de aquellos mundos de ficción o al mero placer de consumo (Van Looy y Baetens, 2003; Sánchez-Mesa, 2011, 2012; Pérez Latorre, 2012). La posición de M.-L Ryan aquí es especial: a pesar de confluir en un concepto abstracto de «lo narrativo» (que abordamos en el nuevo artículo «On Demediation», de Baetens y Sánchez-Mesa, en prensa), insiste en la necesidad de no olvidar la especificidad mediática (Ryan y Thön, 2014; Ryan, 2016).

${ }^{8}$ Existen por supuesto muchas otras propuestas para organizar el campo de estudio. Una particularmente conocida e igualmente simple (aunque tal vez «menos» radicalmente simple que la que aspiramos a proporcionar aquí) es la ofrecida en Rajewski (2005).

${ }^{9}$ Conviene recordar aquí el concepto de «intermedia» de Dick Higgins y su convicción de que «muchas de las mejores obras que se producen hoy caen entre los medios» (1966: 18). Su entendimiento del término se ha hecho relevante en el intento de distinguirlo respecto a los de mixed media y multimedia. Mientras que intermedia aludiría a la fusión conceptual de los materiales propios de distintas formas de arte, «mix media» o «multimedia» se mantendrían más en el nivel de la yuxtaposición. 
La influencia de esta noción sobre las teorías del relato y la narrativa pueden notarse, particularmente en Ryan y Grishakova (2010), así como en la teoría sobre la transmedialidad orientada a la práctica de la producción de Christy Dena (2009), quien habla de transmodality cuando dos regímenes mediáticos están implicados: el narrativo y el de los juegos ${ }^{10}$. La postura más radical sobre la intermedialidad que defendemos aquí tiende a reclamar que incluso los signos monomodales están caracterizados por una complejidad interna.

La intermedialidad, en definitiva, no es solo un término general que define las relaciones entre medios autónomos, sino que es también el término que identifica la pluralidad interna de cada medio e incluso, yendo un paso más allá, la mera condición de posibilidad de existencia de cualquier medio.

2. 2. La transmedialidad: En lo que respecta al otro término clave, la transmedialidad, sugerimos usarlo para referirse al hecho de que cada vez más obras tienden a aparecer en varios medios. La palabra clave en esta descripción de la transmedialidad es «tienden a»: la reaparición (extensión, reescritura) de una obra en otro medio es una posibilidad, no una regla general; lo que es más, su presencia e impacto depende de su contexto histórico. Este proceso es más evidente en la cibercultura, donde se dan cita una serie de factores: la digitalización masiva de los textos culturales junto a la extensión global del lenguaje de los nuevos medios (Manovich, 2001); una fuerte competición entre las grandes corporaciones de medios de entretenimiento en búsqueda de la más rentable migración crossmedia posible de contenidos, fundamentalmente de personajes y mundos ficcionales (storyworlds); la proliferación de las plataformas de distribución y recepción de la cultura popular y el aumento de la intervención creativa de las nuevas audiencias en este paisaje cultural emergente (del fenómeno fan a los prosumidores). Varias formas de transmedialidad concurren en un contexto creciente de movilidad mediática donde todas las formas de adaptación, reciclaje y extensión se combinan. En ciertos periodos, culturales, entornos, géneros, etc., la transmedialidad estará más presente que en otras. En principio, no obstante, la transmedialidad es un fenómeno universal. Como en el caso de la intermedialidad, que es tanto interna como externa, es necesario hacer una distinción ulterior entre dos aspectos o, si queremos, fases de la transmedialidad.

Por un lado, la transmedialización es el mecanismo o proceso que «adapta» una obra que existe en un medio determinado a otro medio. Podríamos llamar al resultado de estas operaciones como «mundos narrativos transmediales del tipo bola de nieve» (el célebre y muy estudiado caso de Star Wars sería un ejemplo de cómo una gran franquicia de Hollywood se convierte en transmedia). Este proceso, tal y como lo conocemos, no es unidireccional (un libro puede adaptarse en una película, pero también una película puede ser «novelizada» en un libro). No obstante, es necesario subrayar que la transmedialización y la adaptación no son prácticas o fenómenos sinónimos o totalmente equivalentes. $\mathrm{Si}$ bien todas las transmedializaciones son adaptaciones, no todas las adaptaciones son transmedializaciones puesto que es perfectamente posible adaptar una obra manteniéndose en un mismo medio (los ejemplos de adaptación intramedial son incontables, desde las traducciones, a los

\footnotetext{
${ }^{10}$ Dena se apoya en el concepto de «multimodalidad» de Lress y Van Leeuwen (1996).
} 
reader's digest, la reescrituras, parodias, etc., todas ellas casos de adaptaciones no transmediales). Manejamos, como puede intuirse, un concepto más amplio de adaptación del que suele predominar en Estudios de Medios y en la Literatura Comparada, inspirados por el concepto descriptivo de traducción como transfer o interferencia cultural desarrollado por la teoría de los polisistemas y en sintonía con otras propuestas igualmente atentas a una comprensión cultural e histórica de la enorme variedad de prácticas adaptativas y a su relevancia creciente en el entorno progresivamente dominado por los new media (Hutcheon y O’Flynn: 2015). Hay razones, por tanto, para mantener una distinción nítida entre al amplio dominio de la adaptación, entendida aquí como parte del campo de estudio propio de la teoría de la traducción, y el campo más estrecho de la reescritura (Pérez Bowie, 2010).

Por otro lado está también el hecho de que ciertas obras no son primero elaboradas en un determinado medio y luego adaptadas a otro medio distinto, sino que son producidas más o menos simultáneamente en varios medios, ninguno de los cuales resulta ser en realidad la «fuente» de los otros. Y ello a través de un diseño de producción multiplataforma donde un mundo ficcional emerge a través de una compleja planificación de múltiples contribuciones de distintos medios. A esta segunda dimensión o fase de la transmedialidad es a lo que Long llamó «hard transmedia» (Long, 2007), definido originalmente por Henry Jenkins como

A process where integral elements of a fiction get dispersed sistematically across multiple delivery channels for the purpose of creating a unified and coordinated entertainment experience. Ideally, each medium makes its own unique contribution to the unfolding of the story (Jenkins, 2009).

Desde la perspectiva de los productores de relatos transmediales o transmedia storytelling (Bernardo, 2011; Phillips, 2011; Dowd et al., 2013), destacamos la reflexión que está llevando a cabo Robert Pratten, CEO de Transmedia Storyteller, quien en la primera edición de su libro Getting Started in Transmedia Storytelling, ofrecía la siguiente definición:

Transmedia storytelling» is telling a story across multiple media and preferably, although it doesn't always happen, with a degree of audience participation, interaction or collaboration. In transmedia storytelling, engagement with each successive media heightens the audience's understanding of, enjoyment of and affection for the story. To do this successfully, the embodiment of the story in each media needs to be satisfying in its own right while enjoyment of all the media should be greater than the sum of the parts (Pratten, 2011: 1).

Es significativo, cuando se lee, cuatro años después, la nueva definición ofrecida por Pratten en la segunda edición de su libro, el desplazamiento hacia la centralidad de la «experiencia» de los usuarios, donde el Transmedia Storytelling se entiende ya como una «filosofía del diseño de producción», cuyo énfasis no descansa ya tanto en el componente «multiplataforma» como en facilitar «a synergy between the content and a focus on emotional, participatory experience for the audience» (Pratten, 2015: 3). Obviamente, dicho movimiento está relacionado con el legítimo interés industrial y comercial del discurso de Pratten.

Desde un punto de vista teórico, resulta crucial marcar la diferencia entre estos dos tipos de transmedialidad, pues si bien son, al mismo tiempo, bastante comparables, ya que ambos son síntomas de lo que Jenkins (2006) llamó «cultura de la convergencia», también son muy distintos, pues el modo 
en que descansan en la adaptación sigue orientaciones diferentes. Si en el primer caso nos encontramos con un empleo de la adaptación en un sentido bastante tradicional (una obra que existe en un determinado medio se convierte en otra cosa en un medio distinto), en el segundo, si bien no se carece por supuesto de una «fuente», se arranca más bien de lo que podemos llamar un «contenido demediado». La noción de demediación —en clara referencia a la de remediación de Bolter y Grusin (1999) - ha sido planteada por Garrett Stewart (2011) para identificar lo que él llama bibliobjets, esto es, libros usados como material escultórico en instalaciones y arte visual. La demediación, sin embargo, es un concepto que va bastante más allá de su uso en los espacios de las galerías o museos. Si bien la palabra misma no aparece directamente en este contexto, es posible encontrar claros ecos en la discusión actual sobre la publicación de libros en la era de la reproducción digital. Tal y como ha demostrado Thompson (2010), tanto los editores modernos multimedia como, los productores transmedia, parecen haber llevado a cabo la sustitución del «texto» por el «contenido» o incluso, añadiríamos nosotros también, por la idea antes citada de la «experiencia». De modo más general, la idea de que los cuerpos o la materia misma desaparecen en el espacio digital, donde solo encontraríamos ideas e información («libre» para circular sin restricciones de carácter material) es uno de los mitos más potentes que sostienen el mundo de la ciber-utopía.

En el campo más especializado de los estudios narratológicos, el éxito actual de enfoques teóricos cognitivistas - aquellos que se centran fundamentalmente en la historia (story) a expensas de lo narrativo en cuanto discurso- puede tener algo que ver con esta ilusión del flujo del significado puro o abstracto (Sánchez-Mesa, 2014). Nuestro reciclaje del término «demediación», que proponemos entender más bien en cuanto proceso («demediatización»), apunta en una dirección distinta.

En primer lugar porque no compartimos la idea del contenido como una entidad inmaterial. Aunque no todas las franquicias crossmediales o los proyectos estrictamente de narrativa transmedial desplazan la historia a un lugar secundario, es evidente la tendencia creciente a priorizar la construcción de los mundos narrativos o storyworlds a partir de la llamada mothership o conjunto de contenidos (personajes, espacios, tiempos, valores) que conforman ese mundo «previo» a las historias que se desarrollan en él. Esta es una tendencia generalizada en el discurso tanto profesional como académico: el propio término «storytelling» se vería desplazado por el de «world building» o por la migración de las licencias o franquicias crossmedia (Aarseth, 2006). La principal teórica de la narratología transmedia, M.-L. Ryan, acuñó el sintagma «transmedial narrative worlds» (Ryan y Thön, 2014) y ahora ratifica ese desplazamiento, coincidiendo con Espen Aarseth en que tal vez el término «storytelling» es una etiqueta imprecisa ${ }^{11}$. Ya en su muy influyente artículo Klastrup y Pajares Tosca definieron los transmedial storyworlds como «abstract content systems from which a repertoire of fictional stories can be actualized or derived across a variety of media forms» (2004: 409). Compartida esa categoría de «worldness», creadores y audiencias o participantes reconocerían esa entidad en la constancia de un mythos («the backstory of all backstorites»), un topos (aquello que se espera de la

\footnotetext{
${ }^{11}$ En sus contribuciones el número 17 de ArtNodes, «Narrativas transmedia», en prensa.
} 
navegación de ese mundo físico) y un ethos (el código de comportamiento y de valores morales de un mundo $)^{12}$. Incluso una teoría de base materialista, bastante reticente a reconocer la dimensión ficcional o imaginaria de los videojuegos, como la ludo-narrativa de Espen Aarseth, descansa en una versión de la narratología y en un concepto de «historia» dependiente de la distinción estructural «kernels» \& «satellites» (Aarseth, 2012: 131), completamente periférica respecto a los personajes y la construcción de mundos. Los personajes, su integridad y movilidad a través de las franquicias cross-genéricas es el factor clave. Robert Pratten, por su parte, citando a Mark P. Wolf y su Building Imaginary Worlds (2012), coincide en esta predominancia de los storyworlds — «the fabric of details that makes a story believable» (Pratten, 2015: 32) — respecto de las historias. En cualquier caso, quizás de forma paradójica, ese productor es uno de los que más recuerda la importancia de la calidad de la dimensión narrativa para lograr el objetivo fundamental de todo proyecto transmedia, que es ser relevante para su audiencia: «I too often see projects with too many characters on too many platforms with too little story. It seems as though some people think that just having lots of stuff going on will be enough but it's the storytelling that's vital to providing the cohesion across the platforms ${ }^{13}$.

Desde una perspectiva sociosemiótica, no existe el significado sin el significante, no se da un interpretante sin signo, etc. Más aún, si consideramos como referente el concepto seminal del signo ideológico de Valentín Voloshinov y de la poética social de Mijaíl Bajtín, no deberíamos descuidar que la articulación formal y discursiva de los contenidos de una obra comportan una evaluación del mundo a través de los personajes y sus lenguajes sociales, que quedan fuera de foco cuando el ethos (importante, hay que reconocerlo, para el discurso de la producción del Transmedia Storytelling) se considera al margen de los distintos modos de enunciación propios de cada medio. Hablamos, no obstante, de tendencias dominantes, pues es evidente que no faltan proyectos que sí tratan de explotar esta dimensión discursiva de las narrativas transmediales, eso sí, en producciones habitualmente al margen de la gran industria, o dentro de los circuitos independientes, caso, por ejemplo, de la narrativa transmedial Plot 28 (A. Serra y J. Hernández, 2008) o del web-doc La primavera rosa (M. de la Torre 2012) $)^{14}$.

Por otro lado, la misma N. Katherine Hayles enfatizó la necesidad de pensar el mundo de los bits y los bytes en cuanto «cultura incorporada», es decir, evitando la disolución de la materialidad y la corporalidad en ideales puramente abstractos o vacíos (Hayles, 1999).

De cualquier manera, usamos aquí el término demediación para referirnos al hecho de que el contenido de una obra no tiene por qué verse «completamente» realizado en un medio antes de que sea posible adaptarlo en otro medio. Esto implica que dicho contenido haya sido elaborado de tal forma que pueda ser fácilmente «reelaborado»o «expandido» en varios medios, sin verse entorpecida dicha migración o expansión por la existencia de una forma preexistente.

\footnotetext{
${ }^{12}$ Para una revisión cabal de las teorías de los mundos transmediales, véase Rosendo (2015).

${ }^{13}$ Entrevista con Robert Pratten de Domingo Sánchez-Mesa (en Sánchez-Mesa; Alberich; Rosendo, eds., en prensa.

${ }^{14}$ http://www.plot28.com; http://www.laprimaverarosa.com.
} 
La razón fundamental para esta demediación no es la digitalización de todos los medios sino más bien el cambio desde las formas culturales tradicionales hacia las industrias culturales y creativas, en las cuales la explotación de una idea dada, o de un argumento o formato en tantas formas y plataformas como sea posible es una necesidad económica. La digitalización refuerza esta tendencia hacia la demediación en tanto transmedialización, pero en realidad no es el detonante fundamental de este cambio. Además, como ha demostrado convincentemente Matthieu Letourneux (2014), no se trata tan solo de la puesta entre paréntesis (parcial) de la forma material del contenido inicial, sino que este proceso también implica una redefinición de más alcance de lo que el contenido realmente es o de la forma específica que puede tomar una vez que se integra en la lógica cultural-industrial de la serialización $^{15}$, una dinámica que no por casualidad fue incluida por Jenkins entre sus siete principios del transmedia storytelling (2009) .

Pensar en la historia como «contenido», por ejemplo, no significa que uno intente inventar una historia que vaya a resistirse lo menos posible a su adaptación en una película, un videojuego, una novela, un cómic, un juguete, un parque temático, etc. Se referirá antes que nada al cambio del eje narrativo desde la historia (en el sentido de una serie de acciones organizadas en la forma de un argumento) al mundo narrativo transmedial (storyworld) ya definido más arriba siguiendo a Klastrup y Pajares Tosca (2004) como ese sistema abstracto de contenidos, contenedor y generador de historias en distintos medios.

Aunque la noción de «transmedia storytelling» cubre claramente los dos aspectos de la transmedialidad abordados aquí (la adaptación en sentido más tradicional por un lado, y la transmedialización del contenido demediado por el otro), las dramáticas implicaciones de esta distinción para la noción misma de relato (storytelling) no siempre son reconocidas. En el primer caso, el transmedia storytelling estará más cercano a la adaptación de la historia, tal y como se da en las franquicias tradicionales. En el último caso, el transmedia storytelling tendrá más que ver con añadir una línea narrativa a un mundo narrativo. En este punto, la dimensión discursiva de la historia, esto es, el foco o perspectiva narrativa, la orquestación de voces y cosmovisiones colectivas, los lenguajes sociales o heteroglosia) resultará menos elaborada con el fin de evitar lo más posible dificultades a la hora de migrar a otros medios.

\footnotetext{
15 Todas las obras que funcionan en un contexto de cultura de masas (libros, films, juegos) dependen menos de la noción de «género» (cuya relevancia no desaparece) como de la noción de «serie» (que es la agrupación de los ítems en conjuntos más grandes); dentro de esos todos, todos los ítems son variaciones del mismo molde, de la misma estructura, del mismo universo). A grandes trazos hay tres tipos distintos de serialización: a) serialización: una obra se publica por entregas (ej.: una serie de TV) y continua mientras hay público dispuesto a comprarla; b) una obra se publica como ítem independiente, pero su éxito hace posible expandirla en nuevas formas en el mismo medio (ej.: series de comics construidas en torno al mismo personajes, o las secuelas y precuelas cinematográficas); c) la expansión de una obra desde un medio a otro (Tintín como comic, pero también como cine de animación, etc.), en teoría una combinación de adaptación y transmedialidad. En este último sentido del término, en el que descansa el horizonte de la serialización contemporánea, la relación entre demediación y serialización se torna clara: la demediación es tanto la condición de la serialización expandida y una consecuencia de ella (de ahí por ejemplo toda la idea del «universo» como el punto de partida de la creación cultural, idea que demedia la obra desde el principio, al tiempo que hace la serialización mucho más fácil).
} 


\section{La Literatura, ¿una «práctica residual»?}

Llega el momento de regresar a la «literatura» en esta revisión de la «literatura comparada» y de examinar su posición, que nosotros estimamos vital, en este nuevo contexto digital convertido hoy en el marco de toda producción cultural (Hayles, 2008). El hecho de que toda forma de producción se encuentre ahora influida y transformada por lo que podemos llamar paradigma digital, no implica, sin embargo, que la literatura y el libro se hayan convertido en dominios residuales de la cultura, cuyas formas dominantes y emergentes (por seguir aquí la terminología acuñada por Raymond Williams, 1977) serían digitales y multimedia.

Podríamos tener la impresión de que la literatura está evolucionando hacia nuevas formas de relato transmedial y esto, en efecto, es así. Estamos ante un giro o cambio decisivo en la escritura contemporánea. No obstante, sería peligroso concluir que esta evolución es lineal o teleológica. En este caso, no hay razón alguna para pensar que la literatura de mañana deberá convertirse en relato transmedial, con solo un espacio marginal para lo que solía considerarse literatura en sus mediaciones tradicionales. Como el mismo Henry Jenkins (2003) dejó claro desde un principio «not all stories will flow across media».

Dos son las razones principales que apoyan nuestra forma de pensar. En primer lugar está la curiosa constatación (al menos según nuestra percepción) de que la conexión entre lo transmedia y el relato (storytelling) está lejos de ser tan natural o evidente como ha llegado a parecer. Después de todo, la literatura más innovadora del último siglo ha tenido, a veces implícitamente y a menudo explícitamente, una fuerte inclinación antinarrativa ${ }^{16}$. La novela modernista anglosajona ha sido acusada de haber abandonado la base misma del relato (Kermode, 2000). Otros movimientos modernistas o bien han ignorado la novela (por ej. el Surrealismo francés) o bien han tratado de erradicar sus pilares básicos, como son el tiempo, el espacio, el personaje, el argumento, etc. (como en el Nouveau Roman). Es verdad que el retorno de la historia y del argumento, tras los numerosos experimentos del siglo XX es, en general, una característica de la escritura contemporánea, si bien este retorno a lo narrativo no implica que toda literatura (y con objeto de alcanzar una mayor claridad nos limitamos aquí a la literatura en prosa) sea por definición narrativa o que el relato sea, igualmente por definición, un rasgo dominante de la literatura. Incluso en una narrativa el énfasis puede ponerse en otros aspectos, convirtiendo la historia en un pretexto para trabajar otras prioridades (como el estilo o la reflexión filosófica, por ejemplo). El énfasis exclusivo en el relato es, por consiguiente, una reducción deplorable de las posibilidades de la literatura, con el subsiguiente énfasis en la demediación que ciertos tipos de relato transmedial desgraciadamente refuerzan (como se ha argumentado más arriba, dicho tipo de relato tiende a reforzar los elementos del mundo narrativo más que el relato mismo, quedando el último reducido a no más que una expansión del primero). Dicho de otro modo: con objeto de explorar más allá sus posibilidades, lo peor que puede hacer la literatura transmedializada

\footnotetext{
${ }^{16}$ Una observación similar podría hacerse del campo de la pintura y de la escultura, con el modernismo de Greenberg aspirando a excluir la historia y la narrativa como no esenciales para la pintura (Greenberg de hecho usa algunas veces el término «literatura» para definir la preocupación de la pintura respecto al asunto de su contenido).
} 
es continuar centrándose exclusivamente en el relato (storytelling). En la práctica, por supuesto esto no es lo que de hecho está ocurriendo, como puede comprobarse, por ejemplo, en las antologías seleccionadas por la $E L O$ (Electronic Literature Organization) y es razonable prever que el estudio teórico de la literatura transmedia se abrirá a formas no narrativas de escritura ${ }^{17}$.

En línea con este primer argumento nos gustaría recordar la crítica ideológica del relato (storytelling) en tanto técnica del marketing que realizara André Salmón (2007), a propósito, por ejemplo, de las campañas políticas. El poder persuasivo de las historias es bien conocido por los académicos de la literatura, que a menudo han subrayado la capacidad de mantener a la audiencia bajo el encanto del narrador («storyteller») como uno de los rasgos más fundamentales de la narrativa. Del mismo modo, como todos los narratólogos saben, la audiencia debe encontrar un interés en la historia que se cuenta, factor que determina el funcionamiento de la misma, y que es tarea del narrador evitar la pregunta «¿y entonces qué?». Hasta cierto punto podríamos preguntarnos si no hay una convergencia histórica profunda (sic) entre la apropiación del transmedia storytelling por las industrias culturales actuales y el abrazo general de la condición post-mediática por parte de los conglomerados multimedia en los años 90 (Krauss, 1999). En ambos casos el objetivo industrial-comercial básico es promover nuevas formas de contenido (demediado) que solo es accesible a través de la compra de nuevos equipos (multimedia).

En segundo lugar, tenemos que reconocer también que la emergencia de la cultura digital no es solo un fenómeno que reemplaza a formas de escritura más viejas, no digitales, con formas de escritura más nuevas, digitales y multimedia, sino que también produce un mecanismo de readaptación, un mecanismo que Bolter y Grusin (1999) llaman repurposing ${ }^{18}$ : un medio «viejo» (en este caso la literatura) reacciona a un medio nuevo (la escritura multimedia digital), incorporando elementos de este nuevo medio o, de forma más radical, destacando elementos que son específicos del medio, es decir, elementos que resisten la transmedialización ${ }^{19}$. Aunque Bolter y Grusin no entran a explorar este dominio, es esto precisamente lo que muchos artistas hacen, tanto en literatura como en otras artes no verbales.

\section{Una galería de géneros intermediales}

En la parte final de este ensayo, nos gustaría presentar brevemente algunos ejemplos seleccionados de prácticas literarias y culturales diversas que sugieren la reinvención creativa de la escritura literaria en el medio ambiente digital contemporáneo. Dichos ejemplos están tomados de cinco campos o subcampos y, por supuesto, ofrecen una muestra nada exhaustiva pero esperamos que

\footnotetext{
${ }^{17}$ Esta fue parte de la propuesta de panel presentado por el proyecto Nar-Trans en la Internacional Conference «The End(s) of Literature», Bergen 5-8 agosto 2015, así como de las ponencias de Rui Torres en los seminarios internacionales de Granada (2015 y 2016).

${ }^{18}$ El término podría ser traducido como «replicación» en el sentido que supone asumir un rasgo del medio que se está adaptando, reorientando alguno de sus efectos de sentido, al tiempo que apropiándose de otros.

${ }^{19} \mathrm{La}$ especificidad mediática, por supuesto, no equivale a la pureza: no pocas visiones contemporáneas de aquella contemplan tanto una diversidad interna como el cambio histórico (Costello, 2008).
} 
lo más representativa posible de dichos dominios de creación. Lo que nos interesa aquí es precisamente la amplitud y el alcance de los dominios a los que apuntan estos casos de estudio, así como la complejidad con la que se articulan y combinan los distintos sentidos de la intermedialidad y la transmedialidad definidos en la primera parte del texto.

4. 1. Los ejemplos más obvios que sobrepasan al relato (storytelling) en el sentido más trivial de la palabra (esto es, en cuanto continuación narrativa de un storyworld demediatizado) pueden encontrarse en la poesía. No solo porque la poesía insiste en otras dimensiones distintas a la narrativa, lo que es una obviedad en la literatura modernista, sino también porque se opone fuertemente a las ideas de demediatización y desmaterialización. La poesía digital es, con mucha frecuencia, extremadamente específica en lo mediático. Torres y Block identifican en la autorreferencialidad (material y mediática) una de las notas específicas de la poesía digital (2007). Descansa en rasgos que son originalmente digitales y que son difíciles de transferir directamente desde la pantalla a la página; por supuesto siempre se puede transmedializar una obra nativa digital en un medio impreso, pero esta operación no es ya un simple transfer, sino la producción de una obra completamente nueva, como puede verse por ejemplo en Tokyo de Eric Sadin, quien adapta y reescribe en forma de libro un hipertexto visual, After Tokyo. Además, críticos culturales como Peter Lunenfeld (2001) han sugerido que la literatura digital ha puesto en primer plano de forma paradójica elementos que habían sido silenciados por la imprenta, tales como la presencia corporal y la voz del autor real. No en vano la poesía digital tiende a evolucionar hacia una poesía de la performance, con el autor presente y estableciendo un diálogo creativo con el software y el hardware de su creación digital ${ }^{20}$. Yendo un paso más allá, podríamos destacar la presencia, tanto cuantitativa como cualitativa, de la poesía (impresa) en el entorno digital y de las narrativas transmediales. Un buen ejemplo de ello es $E l$ Cosmonauta, película dirigida por Nicolás Alcalá (2013) ${ }^{21}$, un título notable no solo por su apuesta por un sistema de producción basado en el crowdfunding y licencia Creative Commons, sino por el papel de la poesía en su entramado ficcional (la obra está parcialmente inspirada en un libro de poemas de Henry Pierrot, Poética para Cosmonautas, 2005), la especificidad de la estética de su intertexto principal (el cine de Tarkovsky), la película misma (en la que la banda sonora no está exenta de elementos poéticos) y la arquitectura y el diseño de una experiencia transmedial (webisodes, fiestas y encuentros, construcción de una comunidad fan a través de las redes sociales y la participación recreativa a través del remixing, una película, el merchandising) que hacen de El Cosmonauta (film) el centro de un universo transmedial en el que la poesía (impresa) juega un papel relevante.

4. 2. Un segundo grupo de ejemplos tiene que ver menos con la integración -y la persistenciade la literatura impresa en entornos transmediales que con el desarrollo de nuevas formas de producción literaria que combinan, por un lado, aspectos mediáticos específicos de la escritura y la

\footnotetext{
${ }^{20}$ Esto es lo que se ha dado en llamar el «demo or die» (Lunenfeld, 2001, comentado en Baetens y Van Looy, 2008).

${ }^{21}$ http://es.cosmonautexperience.com.
} 
lectura en modo impreso y, por otro, una conciencia dispuesta a desarrollar precisamente en formato impreso algunas de las preguntas planteadas por el giro digital mismo. House of Leaves (2000) de Mark Danielewsky, Tree of Codes (2010) de Jonathan Safran Foer's o incluso S. (2013) de Doug Dort y, ni más ni menos, que el productor de Hollywood J. J. Abrahams, son ejemplos bien recientes de un movimiento que podría llamarse «back to the book» ${ }^{22}$, si bien la creencia de que la cultura impresa no está tan indefensa, por así decirlo, respecto al giro digital y de que se encuentra incluso bien equipada para explorar las muchas posibilidades abiertas no resulta ya más una posición marginal o reaccionaria ${ }^{23}$. Una variación interesante de este principio de «vuelta al libro» puede observarse también en el campo de la literatura gráfica, más concretamente en el florecimiento reciente de la novela gráfica. A pesar de la miríada de exigentes ejemplos y modelos que podemos encontrar hoy en el campo de los cómics y las novelas gráficas digitales, debería destacarse que los creadores más innovadores del campo (pensemos en Chris Ware, Charles Burns o Daniel Clowes) prefieren continuar trabajando en formato impreso más que indagar en las 1001 posibilidades que ofrece lo digital. Y esta resistencia no se explica simplemente por la formación visual que tienen la mayoría de estos artistas, todos ellos graduados en escuelas de arte, o por su fascinación fetichista por el papel y la tinta. No menos importante es su conciencia de que el formato impreso está mejor equipado que la pantalla para explorar los rasgos más distintivos de la narrativa gráfica en los cómics y la novela gráfica: la combinación de la secuencialidad — un panel detrás de otro— y la simultaneidad — la página como una composición unificada ${ }^{24}$.

4. 3. Otra categoría de ejemplos puede encontrarse en el contexto de lo que inicialmente se llamó «escritura conceptual» (Dworkin y Goldsmith, 2011) y ahora escritura «no creativa» (Goldsmith, 2011; Perloff, 2011). Influenciada por una amplia variedad de movimientos artísticos del siglo XX (por ejemplo el ready-made art, la escritura troceada — cut up - de Burrough's, o el détournement situacionista), el uso creativo del fragmento (sampling), la copia y el plagio han sido aumentados y acelerados por la emergencia de las herramientas digitales en la literatura. Aquí también, no obstante, la idea de que gracias al ordenador personal el escritor puede empezar a hacer ahora lo que los artistas visuales pudieron hacer tras la invención de la fotografía (esto es, reproducir creativamente el mundo en vez de copiarlo de una forma artesanal) no ha llevado a un final a la cultura impresa, más bien lo contrario. Un buen ejemplo de ello es el remake de la colección de cuentos de Borges El Hacedor (1960) de Agustín Fernández Mallo, sintomáticamente subtitulado, Remake (2011) ${ }^{25}$. En su versión de

\footnotetext{
${ }^{22}$ Este es el título de un programa de investigación dirigido por Kiene Brillenburg Wurth en la Universidad de Utrecht.

${ }^{23}$ En el contexto español la tendencia conocida como «afterpop» (siguiendo el ensayo del mismo título de Eloy Fernández Porta, 2010) o «generación mutante», puede ser considerda desde esta instancia, sobre todo en casos como el de Fernández Mallo (Nocilla Project), Juan F. Ferré (Providence, Karnaval), Jorge Carrión (Las Huellas) o Vicente L. Mora (Alba Crom), cuyo ensayo El lectoespectador (2012) es una buena revisión para de las estrategias de remediación y adaptación de la escritura literaria del siglo XXI.

${ }^{24}$ Acerca de estas cuestiones véase Groensteen (2013), Miodrag (2013) o Chute y Jagoda (2014).

${ }^{25}$ El caso de este libro es casi mítico en la cultura digital en lengua española al haber sido retirado del mercado (editorial Alfaguara), en septiembre de 2011, ante la demanda por plagio del abogado de la viuda de Borges, María Kodama. Véase http://cultura.elpais.com/cultura/2011/10/01/actualidad/1317420001_850215.html.
} 
El Hacedor, Fernández Mallo ofrece una especie de «cover» u homenaje (más o menos siguiendo el espíritu de las versiones homenaje en la música popular). Aunque la obra se encuentra animada sin duda por el Zeitgeist cultural de la reproducción creativa (¡no olvidemos que Borges era un maestro y sublime ejemplo de dicho espíritu!) la escritura de Mallo habría perdido buena parte de su agudeza si se hubiera producido online, a modo por ejemplo de una fan fiction. En este sentido es muy ilustrativo del convencimiento de muchos escritores contemporáneos de que tiene sentido seguir trabajando en formato impreso. En dicho libro, el tríptico titulado «Mutaciones» condensa muy bien una serie de motivos literarios (radicalmente borgesianos) tales como el «doble», «el tiempo circular» o la «cartografía» en tanto metonimia de la mediación verbal literaria. Pero lo específicamente transmedial en este relato es la remediación o adaptación operada del software Google Earth, al mismo tiempo que se transmedializa tanto Internet como el libro de fotografías que da pie al relato (ya de por sí intermedial) - aunque en este caso de una forma limitada - a través de una serie de enlaces a YouTube y a otras redes sociales, operación que expande la narrativa literaria original. La (con)fusión de una aparente réplica «física» de la visita fotográfica de Robert Smithson (padre del Land Art) a Passaic (New Jersey) y la reescritura virtual de dicho libro a través de Google Earth, de la cámara del iPhone del narrador o de su iPad, señala algunas de las posibilidades literarias de la remediación literaria (impresa) de los nuevos medios así como escenifica la oscilación permanente entre la intermedialidad y la práctica convergente de la transmedialidad.

4. 4. Un cuarto nicho en el que hay espacio para esta extensión de la literatura es el del documental web o $i$-doc (interactive documentary), un modo interactivo de no ficción que ha irrumpido con gran pujanza, moviéndose en la encrucijada de sus raíces, por un lado, en las obras cinemáticas vanguardistas de Dziga Vertov, Chris Marker o en las sinfonías fílmicas de Jean Vigo o Walter Ruttmann y, por el otro, en la estética de los nuevos medios definida a partir de la base de datos por Lev Manovich (2001) y las opciones de Internet como gran laboratorio de publicación, distribución, circulación y participación de sus audiencias. Documentales como Highrise: Out of my Window (Caterina Cizek, NFB, 2010) ${ }^{26}$ expande una serie de cortometrajes documentales a modo de collage urbano hacia una narrativa cibertextual, multiplicando el viaje virtual alrededor del mundo del lectorespectador-usuario (de Johannesburg a La Habana; desde Chicago a Phnom-Penh) a través de testimonios de ciudadanos que habitan en rascacielos de las ciudades más populosas. Además de la navegación gracias al mapa de rascacielos (recordemos que la navegación es el segundo principio clave de la estética de los nuevos medios según Manovich), los internautas pueden subir sus propias fotos a Flickr, quedando habilitado así otro modo de intervención participativa dentro de esta cosmovisión que proporciona la pieza. Semejante exploración, inteligente y compleja, en las narrativas factuales encuentra un énfasis de gran potencia inmersiva en el caso de Thanatorama (del grupo multimedia francés Upian, 2008) ${ }^{27}$, autodefinido como «une aventure dont vous êtes le héros mort». A medida que

\footnotetext{
${ }^{26} \mathrm{http}: / /$ highrise.nfb.ca, winner of the International Digital Emmy for Non-Fiction 2011.

${ }^{27}$ http://www.thanatorama.com.
} 
elegimos distintos caminos en la estructura narrativa hipermedia, un narrador en segunda persona introduce al finado en los distintos procesos que tienen lugar una vez que nos unimos al reino de los difuntos (certificación de la muerte, contrato para elegir entre el entierro o la cremación, embalsamiento del cadáver, etc.) incluyendo la experiencia de familiarizarse con los profesionales de la muerte (conductores y empresarios de funerarias, embalsamadores, enterradores, etc.).

En el contexto hispánico, el género está experimentando un desarrollo sobresaliente en España, con laboratorios como el de RTVE, (Las sin sombrero, 2015 es una pieza interactiva sobre las mujeres de la Generación del 27) ${ }^{28}$ o de la mano de creadores e investigadores como Arnau Gifreu (coordinador de proyectos como InterDocs Barcelona o Come/In/Doc) ${ }^{29}$, si bien los desarrollos más notables están teniendo lugar en Latinoamérica con multitud de proyectos entre los que podemos citar los argentinos «Proyecto Walsh» and «Malvinas30» de Álvaro Liuzzi ${ }^{30}$; «Calles Perdidas» or «Mujeres en Venta» ${ }^{31}$ de Fernando Irigaray (Universidad Nacional de Rosario); el proyecto peruano Quipu ${ }^{32}$, o el colombiano enModoP $P^{33}$.

Fue Jay D. Bolter (1991) quien introdujo la metáfora de los writing spaces para referirse a la centralidad del espacio en los procesos de remediación de los nuevos medios. Las narrativas inmersivas (pervasive narratives) refuerzan esta tendencia, explotando las oportunidades que los softwares e interfaces de geolocalización (GPS), los juegos de realidad alternativa (ARGs) con clásicos como «The Beast» (realizado para el film IA de S. Spielberg, 2001) o el relato construido en torno a The Blair Witch Project (D. Myrick y E. Sánchez, 1999), juegos que representan bien el tipo de formato «integrado» de una experiencia transmedial según el productor británico Robert Pratten (2015: 16). A dichos «writing spaces» se unirían, un paso más allá, los generados a través de la escritura y diseño de experiencias de Realidad Alternativa (AR) como el ficcionalizado en el film de D. Fincher The Game (1997). En comparación el nuevo boom de las tecnologías y productos de Realidad Virtual (VR), donde la tecnología «engaña» al ojo y al cerebro humano generando un sofisticado y high-tech trompe d'oeil, aquellas otras experiencias, mucho más basadas en el concepto y técnicas de la performance, están más basadas en la imaginación humana, como dice Pratten (2015: 1999): «it is about human experience in the broadest possible sense because the goal is to achieve an active belief in a parallel world that's interwoven with our everyday world. Nobody's fooled; rather they`re given permission to believe». Con una base literaria claramente anclada en la tradición literaria de la novela de detectives y, en concreto, las de Conan Doyle, Lance Weiler y el equipo del Digital Storytelling Lab de la Universidad de Columbia están desarrollando un macroproyecto de expansión colaborativa, a nivel global, sobre el universo ficcional de las novelas de Sherlock Holmes, basado en los principios y filosofía del llamado «Internet of things» (Weiler, 2016). Los objetos, tratados como «personajes», una vez interconectados

\footnotetext{
${ }^{28}$ http://www.rtve.es/lassinsombrero/es; http://lab.rtve.es/montelab/.

29 http://www.agifreu.com.

30 http://proyectowalsh.com.ar; http://www.malvinastreinta.com.ar.

${ }^{31} \mathrm{http} / /$ www.documedia.com.ar/callesperdidas/; http://www.documedia.com.ar/mujeres/universotransmedia.html.

32 http://blog.quipu-project.com.

${ }^{33} \mathrm{http}$ ://www.enmodop.com/node/146.
} 
con los lectores-jugadores en situaciones de «location based storytelling» se convierten, según R. Pratten (2015: 129), en otra plataforma más de conexión entre la realidad física y la digital, aprovechando el IoT como «la tendencia a la conexión de los objetos cotidianos a Internet».

En cualquier caso, es evidente que nos encontramos con un conglomerado de formatos y tecnologías de relato transmedial que explotan la fascinación contemporánea de la frontera «invisible» (pero no inexistente) entre realidad y ficción, en los sentidos apuntados por Françoise Lavocat en Fait et fiction (2016).

4. 5. Por último pero no menos importante, nos encontramos al final de nuestro paseo a lo largo de los pasajes o no-lugares de esta literatura extendida digitalmente con lo que llamamos precisamente literatura digital o electrónica (e-literature), un conjunto heterogéneo de prácticas, originalmente categorizada, entre otros, por Katherine Hayles y la E.L.O. a través de una ontología digitalmente restrictiva: la literatura digital es aquella que ha sido creada-programada, publicada, distribuida y consumida en dispositivos-pantallas digitales (Hayles, 2008: 1). Tal y como reza el título del libro de Lev Manovich (2013), «Software takes command» a medida que el diseño, la producción, la distribución, la implementación, la recepción y la interacción en torno a los productos culturales tienen lugar a través de dispositivos informáticos. Un espécimen hermoso de la e-literature es In absentia, obra de J. R. Carpenter (2008), una proyecto web de crítica sobre el proceso de aburguesamiento (gentrification) de Montreal. Esta obra es un buen ejemplo de los modos en que las nuevas formas de arte digital narrativo (en este caso hipertextual) consiguen proponer soluciones que incluyen la escritura $^{34}$. Esta es la descripción del proyecto que hace la propia J. R. Carpenter:

It used short «postcard story» narratives and the Google Maps API to address issues of gentrification and its erasures in the Mile End neighborhood of Montreal. The piece was commissioned by an artist-runcentre based in the neighborhood. There are stories in English and in French, written by multiple authors. The launch party was a neighborhood block party held on the Quebec national holiday — thousands of people attended - an animated version of the piece was projected on the underside of a viaduct throughout the event. Stories were added over the course of the summer and into the autumn of that year..$^{35}$

In absentia ilustra amablemente una tendencia dentro de la literatura digital que resiste la idea de romper la historia en muchas pequeñas lexías que el lector tendría que recomponer por sí mismo. En vez de eso, notamos una fuerte propensión hacia una narratividad más fuerte de las lexías: cada lexía propone una especie de cuento o short story (ficcional) en la que la autora da voz al miedo al desahucio de los humildes vecinos. Estas historias están directamente ligadas al contexto no verbal y abierto de la interpretación de los lectores: las historias no deberían leerse como representaciones de algo que ha pasado, sino de una experiencia en marcha cuyo final aún ha de descubrirse. Lo más importante, sin embargo, es el hecho de que este i-doc ficcional limita a propósito el uso del «botón de enlace» («clicking through»): aunque por supuesto el lector es invitado a visitar más de una página

\footnotetext{
${ }^{34}$ Véase http://collection.eliterature.org/2/works/carpenter_in_absentia/ (obra incluida en el vol. 2 de la antología de la Electronic Literature Organization).

${ }^{35}$ E-mail personal a Jan Baetens, 16 de diciembre de 2011.
} 
o sección, el trabajo se construye de tal manera que su (momentánea) interrupción no impide al lector alcanzar un entendimiento del todo. En este sentido, la obra invita a lectores y co-autores a mantener ciertas formas clásicas de la narrativa, en lugar de a jugar con las numerosas posibilidades tecnológicas de las nuevas herramientas de escritura.

Unos años antes, Nick Monfort y Scott Rettberg, en la estela del ala más experimental de la literatura electrónica, crearon Implementation $(2004)^{36}$, una obra que ellos mismos definían del siguiente modo:

Implementation is a novel about psychological warfare, American imperialism, sex, terror, identity, and the idea of place, a project that borrows from the traditions of net.art, mail art, sticker art, conceptual art, situationist theater, serial fiction, and guerilla viral marketing. The text was written collaboratively by Nick Montfort and Scott Rettberg with some contributions from others. Its initial incarnation is as a serial novel printed on sheets of stickers that were distributed in monthly installments beginning in January 2004.

La obra podía leerse de distintas maneras: bien por entregas o «capítulos» (las «hojas» distribuidas como pegatinas que los lectores recibían y debían ubicar en una localización urbana), bien como una novela, si llegabas a disponer de todas las hojas, para lo cual había que intervenir «jugando» pues era preciso enviar las fotos de cada hoja-pegatina ya colocada para conseguir la siguiente hoja y seguir leyendo. Como un «todo», además, la novela acabaría siendo publicada como libro (2012). Aparte de este componente lúdico la lectura se producía en función de una clara serialidad y en tanto proceso estructurado. Existían los lectores de las hojas enviadas por correo, los lectores de la web (donde las fotografías eran fundamentales), los lectores de las pegatinas en la calle, las audiencias de las performances que se hicieron durante el proceso, los lectores participantes-fotógrafos que pegaban las hojas y enviaban las fotos, sin olvidar algunos lectores «traductores», siguiendo otra invitación de los autores. Desde una perspectiva teórica, podemos relacionar Implementation, en tanto narrativa distribuida, tal y como la denomina Jill Walker (2004), con una forma literaria de transmedialidad.

Declaradamente catalogado como una narrativa transmedial, Plot 28 es un experimento probablemente único en el panorama español de la producción transmedial. Con un equipo detrás coordinado por Javier Hernández y Agustín Serra, el proyecto (que se desarrolla desde 2008 hasta hoy) tiene el interés específico para nosotros de colocar a la literatura en un lugar destacado dentro del diseño y la arquitectura de este universo transmedia producido a partir del denominado formato de «Ficción Total». Sus creadores lo definen como:

Una nueva manera de navegar por la ficción a través de múltiples géneros, dispositivos narrativos, juegos y desafíos. Novelas navegables, documentales de creación, blogs, webs, redes sociales, cómics, teatro filmado, música... conforman una gran ficción expansiva total para el interactor contemporáneo. ${ }^{37}$

El proyecto puede catalogarse también dentro del llamado «activismo transmedia», es decir, de un tipo de producciones independientes, animadas por un interés de intervención política y movilización ciudadana en torno a problemas propios de la actual coyuntura de crisis a nivel mundial.

\footnotetext{
${ }^{36} \mathrm{http} / / /$ nickm.com/montfort_rettberg/implementation/.

${ }^{37}$ http://www.plot28.com.
} 
En este caso, se aborda la corrupción política asociada a la especulación financiera e inmobiliaria, llevando al público de este (multi)relato a participar en un complejo proceso de investigación en torno a la misteriosa muerte de un campesino, Damián Forcés, propietario de la última parcela (Plot 28) expropiada en los terrenos en los que se levantó la Expo del Agua en Zaragoza en 2008, un año, por lo demás, que marca el estallido del sistema capitalista financiero con la quiebra de Lehman Brothers, el subsiguiente colapso bursátil y la crisis global económica, social y política subsiguiente. Abierto con un falso documental (estrenado en febrero de 2008) y enmarcado en la web del proyecto como matriz de acceso a las distintas plataformas y medios en los que se distribuye el universo ficcional de Plot 28, la literatura adquirió enseguida un protagonismo central en el despliegue del proyecto, primero a través de la «novela navegable»Plot 28, escrita y producida en formato iPad (BBD 2013), enlazada con toda una serie de documentos con funciones específicas en el relato de las tramas (bioclips de los personajes, podcasts, blogs, webs, redes sociales, etc.). Más tarde con la publicación del libro de relatos Bitácora a la deriva (Javier Hernández, 2015), un texto impreso «enriquecido» con códigos QR, enlaces e invitaciones a twittear que convierten la lectura en una experiencia multiplataforma, ergódica y participativa. El corpus literario, integrado por Plot28. Novela navegable (primero diseñada como app y más tarde como ibook), la Bitácora a la deriva (libro impreso de relatos) y el Diario de Laura (ibook, integrado primero en la novela navegable y luego publicado de forma independiente) conforman el entramado narrativo más complejo y trabajado de este universo. Los textos de esta trilogía están interrelacionados por temas, tramas y personajes. Asimismo, un interfaz específico permite, tanto en la versión app como en los ibooks respectivos, conectar indistintamente el espaciotiempo literario de la novela navegable (iPad) y los textos luego publicados en formato impreso de Bitácora a la deriva, a través de un toque de pantalla en un icono bautizado como «agujero de gusano», una estrategia editorial que desarrolla las posibilidades del hipertexto simulando un sentido de la profundidad («depth links») muy característico de la ficción posmoderna, poniendo así en relación convergencias temáticas, argumentales o de personajes entre ambos textos. Javier Hernández, guionista y creador del diseño transmedial de este universo, siguió muy conscientemente los principios del TS de Henry Jenkins procurando que cada medio o plataforma contribuyera al conjunto en función de su propia especificidad mediática. Así, por ejemplo, la novela gráfica desvela las circunstancias de una subtrama importante, el asesinato de Agustín Serra, productor del falso documental con que se abre el storyline del universo; las webs y los blogs proporcionan líneas narrativas que se expanden o profundizan en las motivaciones de los personajes y el contexto sociopolítico de la ficción transmedial y, por último, los juegos o elementos lúdicos plantean a los seguidores de esta ficción la oportunidad de introducirse en las «interrogantes» del misterio, compilando «pruebas» y contribuyendo con sus propias creaciones («plotys») a la extensión de dicho universo. El componente activista del proyecto llegó a incorporarse en la creación del «grupo 28» (acciones en la red) y del Comando 28 (alguna de cuyas acciones offline llegó incluso a tener secuelas de tipo jurídico). 
En este breve recorrido, el lector seguramente eche de menos otros medios o géneros culturales tan dominantes en la cultura digital como pueden ser la televisión interactiva o los videojuegos. Ambos dominios plantean retos fundamentales a los estudios culturales y comparados de la intermedialidad y la transmedialidad (Sánchez-Mesa, 2012; Ryan y Thön, 2014; Evans 2015; Hutcheon y O’Flynn, 2015). En este sentido, llamamos la atención sobre el trabajo de Nieves Rosendo, cuyos artículos sobre el funcionamiento de la intermedialidad (el fantástico literario) y la transmedialidad en Alan Wake (Rosendo, 2015) o sobre el desarrollo del personaje transmedial como categoría definitoria de un tipo específico de narrativa transmedial, a propósito del caso de la serie de la BBC, Sherlock (Rosendo, en prensa), son tan solo avances de una línea de investigación muy prometedora.

\section{Desde la narrativa al Transmedia Storytelling y de vuelta}

En este artículo, hemos tratado de precisar la posición de la literatura en el campo más amplio de las prácticas culturales transmediales sobre la doble idea de lo narrativo y el relato (storytelling). Antes de nada, este intento nos ha dado la oportunidad de plantear algunas propuestas terminológicas que pueden ayudar a clarificar el sustrato terminológico de los debates en torno a tres nociones inextricablemente relacionadas (intermedial, transmedial, remediación). Estas precisiones terminológicas enfatizan el estatuto singular de la noción de relato/narración (storytelling) en lo que se considera hoy la opción por defecto de cualquier forma de narrativa en las producciones digitales multimedia: el transmedia storytelling. De hecho, da la impresión que, por un lado, la noción de storytelling cubre solo una parte (más o menos pequeña) de la narrativa transmedial, y que, por otro lado, tanto el término-concepto storytelling como el de narrativa se aproximan de forma muy distinta a cuestiones básicas como la adaptación y qué se entiende por medio. Finalmente, hemos presentado una selección de ejemplos del uso, persistencia o incluso invención de formas de literatura y de narrativa literaria dentro de los entornos digitales. Estos ejemplos así como la diversidad de dominios de los cuales son extraídos parecen sugerir que hay espacio para formas renovadas de la narrativa literaria en el mundo del transmedia storytelling.

\section{Bibliografía}

AARSETH, E. (2006): «The Culture and Business of Crossmedia Production», Popular Communication, $4 / 3$, pp. 203-211.

(2012): «A Narrative Theory of Games», FDG '12, Raleigh NC, en https://www.researchgate. net/publication/254006015_A_narrative_theory_of_games (última consulta, 8-12-2016).

Abuín, Anxo (2013): El teatro en el cine. Estudio de una relación intermedial. Madrid, Cátedra.

BOLTER, Jay David, y GRUSIN, Richard (1999): Remediation. Understanding New Media. Cambridge, Mass., MIT Press.

BAetens, Jan (2008): La Novellisation. Du Film au Roman. Bruselas, Les Impressions Nouvelles. (2014): «Le médium n’est pas soluble dans les médias de masse», Hermès, 70, pp. 40-45. 
Baetens, Jan - VAn Looy, Jan (2003): Close Reading New Media. Analyzing Electronic Literature. Lovaina, Leuven University Press.

- (2008) «E-poetry between Image and Performance: a cultural analysis», Journal of E-Media Studies, 1/1, en http://journals.dartmouth.edu/cgi-bin/WebObjects/Journals.woa/xmlpage/4/ article/288 (última consulta, 8-12-2016).

BAETENS, Jan - SÁNCHEZ-MESA, Domingo (en prensa): «On Demediation», Leonardo, con publicación digital anticipada en «The Leonardo Just Accepted (JA) system», MIT Press.

BREA, José Luis (2007): Cultura_RAM. Barcelona, Gedisa.

- (2008): El tercer umbral: el estatuto de las prácticas artísticas en la era del capitalismo cultural. Murcia, Cendeac.

CARPENTER, J. R. (2008): In absentia, en http://collection.eliterature.org/2/works/carpenter_in absentia/about.html (última consulta, 8-12-2016).

Chute, Hillary - JAGODA, Patrick, eds. (2014): Comics \& Media, número especial de Critical Inquiry, $40 / 3$.

COSTELlo, Diarmuid (2008): «On the Very Idea of a "Specific" Medium: Michael Fried and Stanley Cavell on Painting and Photography as Arts», Critical Inquiry, 34/2, pp. 274-312, en https://www2.warwick.ac.uk/fac/soc/philosophy/people/costello/costello_ci_342_fried_cavell. pdf (última consulta, 8-12-2016).

DANIELEWSKI, Mark Z. (2000): House of Leaves. Nueva York, Pantheon.

DenA, Christy (2009) Transmedia Practice; Theorising the Practice of Expressing a Fictional World across Distinct Media and Environments. Sydney, University of Sydney, School of Letters, Art and Media, en http://www.christydena.com/phd/ (última consulta, 8-12-2016).

Dworkin, Craig - GoldSMith, Kenneth, eds. (2011): Against Expression: An Anthology of Conceptual Writing. Chicago, Northwestern University Press.

FERnÁNDEZ MALlo, Agustín (2011): El Hacedor (de Borges). Remake. Madrid, Alfaguara.

FOER, Jonathan Safran (2010): Tree of Codes. Londres, Visual Editions.

GolDSMITH, Kenneth (2011): Uncreative Writing: Managing Language in a Digital Age. Nueva York, Columbia University Press.

Groensteen, Thierry (2013): Comics and Narration. Jackson, The University Press of Mississippi.

GuILlÉN, Claudio (2005): Entre lo uno y lo diverso. Introducción a la literatura comparada (Ayer y hoy). Barcelona, Tusquets ( $1^{\mathrm{a}}$ ed., Barcelona, Crítica, 1985).

Grusin, Richard - Bolter, J. D. (1999): Remediation. Understanding New Media. Cambridge, Mass., MIT Press.

HAYLES, N. Katherine (1999): How We Became Posthuman: Virtual Bodies in Cybernetics, Literature and Informatics. Chicago, Chicago University Press.

- (2008): Electronic Literature. Notre-Dame, Notre-Dame University Press.

HERNÁNDEZ, Javier (2013): Bitácora la deriva. Madrid, Esto no es Berlín. 
HigGins, Dick (1966): Something Else Newsletter, 1/1; reeditado en Horizons. The Poetics and Theory of the Intermedia, Carbondale, Edvardsville, Southern Illinois University Press, 1984.

HutCheOn, L. y O'Flynn, S. (2015): A Theory of Adaptation. Londres / Nueva York, Routledge. JENKINS, Henry (2003): «Transmedia storytelling. Moving characters from books to films to video games can make them stronger and more compelling», MIT Technology Review, en http://www.technologyreview.com/biotech/13052/ (última consulta, 8-12-2016).

(2006): Convergence Culture. Where Old and New Media Collide. Nueva York, New York University Press.

(2009): «The Revenge of the Origami Unicorn: Seven Principles of Transmedia Storytelling», In Confessions of an Aca-Fan (The Official Weblog of Henry Jenkins), 12 de diciembre, en http://henryjenkins.org/2009/12/the_revenge_of_the_origami_uni.html (última consulta, 8-122016).

Kermode, Frank (2000): Sense of an Ending. Studies in the Theory of Fiction (with a New Epilogue). Nueva York, Oxford University Press.

KLASTRUP, Lisbeth - PAJARES TOSCA, Susana (2004): «Transmedial worlds: Rethinking cyberworld design». Proceedings of the International Conference on Cyberworlds IEEEE Computer Society, en http://www.itu.dk/people/klastrup/klastruptosca_transworlds.pdf (última consulta, 8-122016(.

KRAUSS, Rosalind (1999): «A Voyage on the North Sea». Art in the Age of the Post-Medium Condition. Londres, Thames and Hudson.

KRESS, Gunther y VAN LEEUwen, Theo (1996): Reading Images: The Grammar of Visual Design. Nueva York, Routledge.

LAVOCAT, François (2016): Fait et fiction, París, Seuil.

LetourneuX, Matthieu (2014): Penser la sérialité: supports, genres, culture médiatique. HDR, París Ouest La Défense, 6 de diciembre; París, Seuil, en prensa.

Long, Geoffrey (2007): Transmedia Storytelling: Business, Aesthetics and Production at the Jim Henson Company. PhD Diss. Cambridge, Mass., MIT Press.

LunENFELD, Peter (2001): Snap To Grid. Cambridge, Mass., MIT Press.

MANOVICH, Lev (2001): The Languages of New Media. Cambridge, Mass., MIT Press.

(2013): Software Takes Command. Nueva York, Bloomsbury Academic

MCluHAn, Marshall (1964): Understanding Media. Nueva York, McGraw Hill.

MiODRAG, Hannah (2013): Comics and Language. Jackson, The University Press of Mississippi.

Mitchell, William J.T. (2005): «There Are No Visual Media», Journal of Visual Culture, 4/2, pp. 257-266.

Monegal, Antonio (1998): En los límites de la diferencia: poesía en imagen en las vanguardias hispánicas. Barcelona, Tecnos. (2008): «La literatura irreductible», Ínsula: Revista de Letras y Ciencias Humanas, 733-734, pp. 2-5. 
MorA, V. L. (2012): El lectoespectador. Barcelona, Seix Barral

PÉREZ BowIE, José Antonio (2010): Reescrituras fílmicas: Nuevos territorios de la adaptación. Salamanca, Universidad de Salamanca.

PÉREZ LATORRe, Oliver (2012): El lenguaje videolúdico. Análisis de la significación del videojuego. Barcelona, Laertes.

PeRloff, Marjorie (2011): Unoriginal Genius. Chicago: Chicago University Press.

Pierrot, Henry (2013): Poética para Cosmonautas. Madrid, Riot (1 $1^{\mathrm{a}}$ ed. León, Leteo, 2005).

PRATten, R. (2015): Getting Started in Transmedia Storytelling. A practical Guide for Beginners. $2^{\mathrm{a}}$ edición ( $1^{\mathrm{a}}$ ed. online en http://www.tstoryteller.com/getting-started-in-transmedia-storytelling [última consulta, 8-12-2016]).

RAJEWSKY, Irina O. (2005): «Intermediality, Intertextuality, and Remediation: A Literary Perspective on Intermediality», Intermédialités/Intermedialities, 6, pp. 43-64.

RosENDO, Nieves (2015): «Lo fantástico en Alan Wake: remediación, intermedialidad, transmedialidad», Brumal. Revista de Investigación sobre lo Fantástico, 3/1, pp. 73-93.

- (en prensa): «Character-Centered Transmedia Characters. Sherlock Holmes in the 21st. Century», ArtNodes. Revista de Arte, Ciencia y Tecnología, 18.

RYAN, Marie-Laure; (ed.) (2004): Narrative Across Media. The Languages of Storytelling. Lincoln/Londres, University of Nebraska Press.

(2006): Avatars of Story. Minneapolis, University of Minnesota Press.

(en prensa): «Transmedia Narratology \& Transmedia Storytelling», Narrativas Transmediales ArtNodes. Revista de Arte, Ciencia y Tecnología, 18.

RYAN, Marie-Laure - GRISHAKOVA, Marina, eds. (2010): Intermediality and Storytelling. Berlín, Walter de Gruyter.

RYAN, Marie-Laure - THÖN, Noel, eds. (2014): «Storywolds across Media: Introduction», en Storywolds across Media Towards a Media-Conscious Narratology. Lincoln, University of Nebraska Press, pp. 25-49.

SADIN, Eric (2005): Tokyo. París, P. O. L.

SAINT-GELAIS, Richard (2005): «Transfictionality», en David HERMAN et al., eds., The Routledge Encyclopedia of Narrative Theory. Londres, Routledge, pp. 612-613.

SALMON, Christian (2007): Storytelling. La machine à fabriquer des histoires et à formater les esprits. París, La Découverte.

SÁNCHEZ-MESA, Domingo (2011): «Literatura aumentada. Intermedialidad / Transmedialidad o el Viaje de Alicia a través de las pantallas», en Salvador MONTESA, ed., Literatura e Internet. Nuevos Textos, Nuevos Lectores. Málaga, Publicaciones del Congreso de Literatura Española Contemporánea, pp. 109-129.

(2012): «Videojuegos y Cine: intermedialidad / transmedialidad», en Barbara ZECCHI, ed., Teoría y práctica de la adaptacion filmica. Madrid, Editorial de la Universidad Complutense, pp. 205-235. 
(2014): «Transmedia Storytelling: Is It the End of Discourse Theory?», en 1st International Seminar on Transmedial Narratives (Granada, 26-28 de marzo de 2014).

SÁnCHEZ-Mesa, Domingo - AlBerich, J. - Rosendo, N., eds. (en prensa): Narrativas Transmediales. ArtNodes. Revista de Arte, Ciencia y Tecnología, 18.

SCOLARI, Carlos A. (2009): «Transmedia Storytelling: Implicit Consumers, Narrative Worlds and Branding in Contemporary Media Production», International Journal of Communication, 3, pp. 586-606.

(2013): Narrativas transmediales. Cuanto todos los medios cuentan. Bilbao, Deusto.

Stewart, Garrett (2011): Bookwork. Medium to Object to Concept to Art. Chicago, Chicago University Press.

ThOMPSON, John B. (2010): Merchants of Culture. Londres, Polity.

TorRes, Rui y Block, W. F. (2007): «Poetic Transformations in(to) the Digital», en Telepoesis.net (ponencia presentada en la e-Poetry de París, 20-23 mayo).

YÁÑEZ, María (2011): «Informe: documental interactivo. Con la realidad sí se puede jugar», en EMBED.At Audiovisual integrado, http://antes.embed.at/article43.html (última consulta, 8-122016).

WALKER, Jill (2004): «Distributed Narratives: Telling Stories across Networks», presentado en AoIR 5.0. (Brighton 21 de septiembre), en https://www.academia.edu/2182805/Distributed_narrative Telling_stories_across_networks (última consulta, 8-12-2016).

WEILER, L. et al. (2016): Sherlock \& the Internet of Things, en http://www.digitalstorytellinglab.com (última consulta, 8-12-2016).

WILLIAMS, Raymond (1997): Marxism and Literature. Oxford / Nueva York, Oxford University Press. WoLF, Mark J. P. (2012): Building Imaginary Worlds. Nueva York, Routledge. 